Bio -grafía Escritos sobre la Biología y su Enseñanza.

Edición Extra-Ordinaria.

Memorias del 1 Congreso Nacional de Investigación en Enseñanza de la

Biología. VI Encuentro Nacional de Investigación en Enseñanza de la Biología y la Educación Ambiental. ISSN 2027 1034. P. p. 471-478.

\title{
CARACTERIZACIÓN DE LA RELACIÓN ENTRE EL CONOCIMIENTO COTIDIANO Y EL CONOCIMIENTO CIENTÍFICO EN LA CONSTRUCCIÓN DEL CONOCIMIENTO ESCOLAR EN ESTUDIANTES DE 601 DEL IED MIGUEL ANTONIO CARO. PLANTEAMIENTO DEL PROBLEMA
}

\section{POR: Valencia Natalia ${ }^{1} \&$ Elías Amórtegui ${ }^{2}$}

\section{Resumen}

A continuación se presenta el planteamiento del problema de investigación de la practica pedagógica, desarrollada en la IED Miguel Antonio Caro en el marco de la línea de investigación Conocimiento Profesional del Profesor de Ciencias; este proyecto tiene como objetivo identificar la relación entre el conocimiento cotidiano y el conocimiento científico en la construcción del conocimiento escolar en el área de ciencias naturales en los estudiantes del curso 601 de la institución. Específicamente, se describen los fundamentos teóricos centrados en la construcción del conocimiento escolar, algunos trabajos relacionados, y la metodología empleada.

Palabras clave:Conocimiento científico, conocimiento cotidiano, conocimiento escolar.

\section{Abstract}

Bellow is presented the exposition about the problem we can find in teachertraining done in IED Miguel Antonio Caro inside the professional knowledge of science's teacher line of investigation framework. The main objective of this project is to identify the construction of scholar knowledge in Natural Science in sixth level students (601) in relation to the daily and scientific knowledge. Theoretical foundations about the learning construction process, some workshops related to, the methodology and a short advance of first results are described.

\section{Keywords}

Common Knowledge, scientific knowledge, school knowledge

\section{Introducción}

La importancia de la construcción y la definición del conocimiento ha sido tratado y discutido durante mucho tiempo, en donde surgen muchas perspectivas como las empirista y la racionalista, que lo definen muy diferente cada una, desde hechos, datos o información hasta la conciencia o la

\footnotetext{
1 Estudiante Séptimo semestre de Licenciatura en Biología de la Universidad Pedagógica Nacional. practicante del grupo de investigación Conocimiento profesional del profesor de ciencias

2 Magíster en Educación. Profesor de Cátedra del Departamento de Biología de la Universidad Pedagógica Nacional.
} 
Memorias del 1 Congreso Nacional de Investigación en Enseñanza de la

Biología. VI Encuentro Nacional de Investigación en Enseñanza de la Biología y la Educación Ambiental. ISSN 2027 1034. P. p. 471-478.

experiencia, pero en este caso se optará una definición consolidada que plantea el conocimiento como aquel conjunto de saberes prácticos y teóricos que tratan de explicar la realidad del mundo, desde allí se propone la construcción del conocimiento escolar, en este caso en ciencias ya que no solo corresponde construir frente a lo disciplinar (física, química, biología) si no el incluir otros aspectos como, el contexto, ideas y conceptos previos; esto llamado como conocimiento cotidiano, toda esta construcción pretende buscar un aprendizaje significativo de ciencias que permita la comprensión y el desarrollo de las actitudes frente a las ciencias; con el fin de resaltar los componentes en la construcción de conocimiento escolar, este proyecto está enfocado en la construcción de este ya que tiene como objetivo identificar las relaciones entre el conocimiento cotidiano y el conocimiento científico en la construcción del conocimiento escolar en el área de ciencias naturales, desarrollando actividades en la IED Miguel Antonio Caro donde se involucra la docente de ciencias, dando como resultado una aproximación de ideas que presenta este frente a los conocimientos, además caracterizando los elementos que aporte la docente a la construcción de conocimiento escolar en los estudiantes del grupo 601 de la institución .

En el marco de la línea de investigación Conocimiento Profesional del Profesor de Ciencias; este proyecto pretende contribuir a sus problemas de investigación en torno a la identificación y caracterización entre el Conocimiento Disciplinar Científico, el Conocimiento Cotidiano, el Conocimiento Didáctico del Contenido y el Conocimiento Escolar, con el fin de aportar a la clarificación de la construcción de conocimiento que se presenta en la escuela, ya que hay una visión mayoritaria en la cual se cree que en la escuela se construye conocimiento científico, causando así obstáculos para el aprendizaje significativo de las ciencias, este proyecto pretende contribuir a esta aclaración mostrando las relaciones entre los conocimientos (cotidiano, científico, contextual, entre otros), que se presentan en la escuela frente a la enseñanza y el aprendizaje de las ciencias, logrando así una caracterización y reconocimiento del conocimiento escolar, su construcción, su importancia, y las relaciones presentes en esta construcción.

Si bien la construcción del conocimiento escolar es asunto de los docentes, muchos autores se refieren a este conocimiento, de maneras variadas, por ejemplo ciencia escolar, como conocimiento científico escolar (Nardi M. \& Almeida, 2005), conocimiento escolar, contenidos escolares, conocimiento en la escuela, saber escolar, entre otros. Zambrano, A. (2000) presenta una relación entre conocimientos individuales del docente y el estudiante en el contexto de la construcción del conocimiento escolar. Reconociendo la importancia de la construcción del conocimiento escolar se propone investigar ¿Cómo se relaciona el conocimiento cotidiano y el conocimiento científico en la construcción del conocimiento escolar en ciencias naturales, en los estudiantes del curso 601 del ciclo 3, del IED Miguel Antonio Caro? Pretendiendo obtener la importancia de cada uno de los conocimientos que lo conforman y además 
Bio -grafía Escritos sobre la Biología y su Enseñanza.

Edición Extra-Ordinaria.

Memorias del 1 Congreso Nacional de Investigación en Enseñanza de la

Biología. VI Encuentro Nacional de Investigación en Enseñanza de la Biología y la Educación Ambiental. ISSN 2027 1034. P. p. 471-478.

reconociendo el conocimiento escolar como conocimiento resultado de un proceso de aprendizaje significativo en la clase de ciencias.

\section{Marco referencial y conceptual}

Maria Jose Rodrigo (1997) define el conocimiento científico como aquel que trata de mostrar la realidad de las cosas apoyándose en un método científico, es decir con métodos de comprobación, procedimientos e hipótesis, ya que los resultados pueden ser repetibles, cuantificables y observables. Este conocimiento es el fundador de las leyes universales que grandes científicos han propuesto y otros han validado. El conocimiento común es el que los sujetos construyen a partir de vivencias, cultura o tradición, este conocimiento lo construyen todos los sujetos, es autónomo y único, normalmente es transmitido de generación en generación. Y finalmente el conocimiento escolar que es el que se construye a partir de la sustitución complementación o regeneración del conocimiento común tomando como base el conocimiento científico.

Para diferenciar el conocimiento escolar y el conocimiento científico Valbuena (2007) establece que: los científicos investigan lo que quieren aprenden a diferencia de los estudiantes que los obligan aprender contenidos, los científicos se especializan en un área de las ciencias los estudiante deben aprender de todas las áreas, los científicos utilizan todo el tiempo para investigar en ciencia, los estudiantes no solo ven ciencia si no otras áreas de saber que casi siempre están desarticuladas con la ciencia.

El problema en la escuela surge en la separación que presenta el conocimiento disciplinar y el cotidiano, ya que el primero se trabaja en problemas cerrados y es utilizado para superar las evaluaciones, por otra parte el cotidiano es utilizado por los estudiantes para solucionar problemas a diario, además es caracterizado como funcional, útil, permite la solución de problemas, coherente entre otras por esta razón es muy difícil transferir la ciencia trabajada en las clases a la vida cotidiana de los estudiantes. (Valbuena, 2007).

Los docentes deben modificar las dinámicas del trabajo escolar, tendiendo a la integración de conocimientos de diversas fuentes, respetando el conocimiento cotidiano, con el fin de facilitar el aprendizaje significativo y permitir que el explique fácilmente sus propios modelos mentales logrando así la relación entre conocimiento cotidiano y el conocimiento escolar "Es decir, el profesor debe buscar y propiciar situaciones que tengan significativita para los alumnos y poder así aproximarlos a su epistemología [la de ellos], sus representaciones y escenarios cotidianos. La pretensión final es lograr que los estudiantes puedan llegar a diferenciar entre sus conocimientos cotidiano y escolar para así poder enriquecer e interrelacionar dichos conocimientos" (Valbuena, 2007). 
Memorias del 1 Congreso Nacional de Investigación en Enseñanza de la

Biología. VI Encuentro Nacional de Investigación en Enseñanza de la Biología y la Educación Ambiental. ISSN 2027 1034. P. p. 471-478.

Para hablar de construcción de conocimiento es necesario incluir el conocimiento didáctico del contenido (CDC) que se refiere a uno de los conocimiento específicos del conocimiento profesional del profesor que se define como el conocimiento que se necesita para poder transformar los contenidos disciplinares, se construye a partir de la integración de los otros conocimientos con el fin de hacerlos más compresibles a los alumnos y facilitar así su aprendizaje. Se trata entonces de hacer de los contenidos disciplinares, contenidos "enseñables y aprendibles", Bromm (1988) aporta al CDC afirmando que los conocimientos teórico no son simplemente aplicables en el contexto si no que demanda unas transformaciones, en las cuales se permita interrelacionas los conocimientos científicos y los cotidianos.

La construcción del conocimiento escolar desde el Proyecto Curricular Investigación y Renovación Escolar (IRES), no lo define como la sustitución de un conocimiento por otro ni de las características del conocimiento cotidiano y del conocimiento científico por individual, sino que la construcción implica una integración didáctica de estos. La construcción de conocimiento escolar que propone García en 1997, definiendo este como el conocimiento que se propone y se elabora en la escuela, que sea generalizable a otros contextos, la construcción de este conocimiento debe tener en cuenta cuatro apropiaciones:

1. Tener en cuenta el paradigma filosófico que orienta la evolución del conocimiento escolar y hace eje articulador de las restantes hipótesis curriculares, pretendiendo considerar la incompatibilidad entre lo cotidiano y lo científico.

2. Realizar un cambio tratando los problemas socios ambientales del contexto de los sujetos, con el fin de investigar proponer y gestionar proyectos de interés e ir más allá de la distinción entre el conocimiento científico y el escolar.

3. Beneficios del conocimiento científico-técnico en la solución de problemas socio ambientales.

4. Utilizar el conocimiento cotidiano presente en las ideas previas

\section{Metodología}

Esta investigación se ha desarrollado dentro de un enfoque investigativo cualitativo ya que está encaminado al interés por captar la realidad social a través de la percepción que tiene el sujeto en ese contexto (Bryaman, 1988). El propósito es utilizar la observación participativa como técnica donde el investigador, asume un papel dentro del grupo y participar en sus funciones, con el fin de participar para comprender la dinámica del grupo. (Robledo, 2009), esta observación se realizara durante la práctica pedagógica I y II con el grupo 601 del MAC y se utilizara el método análisis de contenido que consiste en la interpretación de textos de una manera sistémica, objetiva 
Bio-grafía Escritos sobre la Biología y su Enseñanza.

Edición Extra-Ordinaria.

Memorias del 1 Congreso Nacional de Investigación en Enseñanza de la

Biología. VI Encuentro Nacional de Investigación en Enseñanza de la Biología y la Educación Ambiental. ISSN 2027 1034. P. p. 471-478.

replicable y valida (Andrue 2001, Piñuel, 2007), en cuanto al diario de campo y documentos obtenidos durante el proceso de enseñanza -aprendizaje con el estudiantes, presentando categorías de análisis que caractericen el conocimiento.

La investigación se desarrollará en 3 fases (Ver Figura 1), la primer fase fue la planeación en la cual se ejecutó la practica I, en donde se realizó todo el planteamiento del problema, se estudió la problemática, se realizó la debida búsqueda del marco de referencia, se diseñaron los instrumentos de recolección de información y se presento una contextualización, en la fase 2 , que inicio en la práctica I y se continuara en la práctica II, en esta fase se aplicaron de los instrumentos de recolección de información (cuestionario y entrevista) además se desarrollo clases en las cuales fuera evidente la relación entre el conocimiento cotidiano y el conocimiento científico. En la fase tres se realizaran el análisis de resultados en el cual se propondrán categorías para la interpretación de resultados obtenidos a través de las notas de campo, los registros audiovisuales, y las clases realizadas por el practicante.

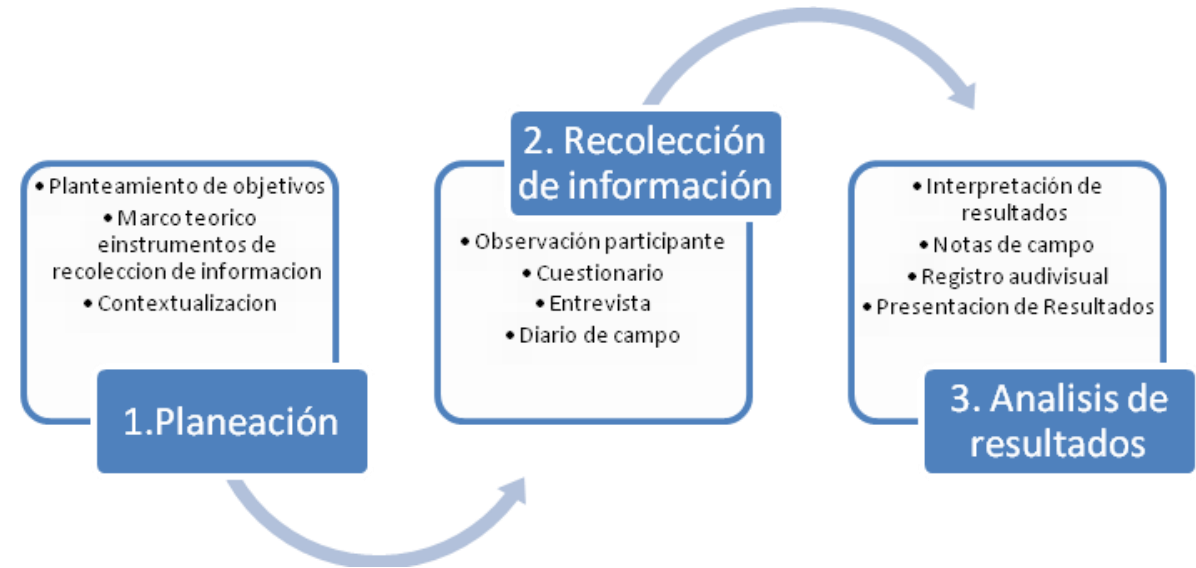

Figura 1 Fases de investigación

Como herramientas para la recolección de información se utilizaron entrevistas semi-estructuradas, y el cuestionario. La entrevista definida por Anger-egg, consiste en una conversación entre dos personas por lo menos, en la cual uno es entrevistador y otro $u$ otros son los entrevistados tiene como objetivo la indagación de información que tenga el entrevistado y es de gran interés para el entrevistador, la entrevista semi-estructurada le permite al entrevistador utilizar las respuestas del entrevistado como más preguntas, y finalmente encontramos la no estructurada que es un dialogo abierto entre los roles de la entrevista. (Perez, 2005). Las preguntas diseñadas en la entrevista pretenden identificar las ideas del maestro frente a la construcción del conocimiento escolar realizando preguntas enfocadas a la indagación al conocimiento profesional del profesor enfocándolo a la caracterización de conocimiento cotidiano y el conocimiento escolar (Ver Tabla 1) 
Bio-grafía Escritos sobre la Biología y su Enseñanza.

Edición Extra-Ordinaria.

Memorias del 1 Congreso Nacional de Investigación en Enseñanza de la

Biología. VI Encuentro Nacional de Investigación en Enseñanza de La Biología y la Educación Ambiental. ISSN 2027 1034. P. p. 471-478.

\begin{tabular}{|c|c|}
\hline Preguntas & Aspecto a indagar \\
\hline $\begin{array}{l}\text { Nombre del docente: } \\
\text { Licenciado en: } \\
\text { Años de experiencia pedagógica: }\end{array}$ & $\begin{array}{l}\text { Estas primera } 3 \text { preguntas se } \\
\text { realizan con el fin de acercarse un } \\
\text { poco frente a la formación del } \\
\text { docente }\end{array}$ \\
\hline $\begin{array}{l}\text { ¿Cuál ha sido su experiencia } \\
\text { como docente? } \\
\text { ¿Cómo se autoevalúa como } \\
\text { docente? ¿Está usted en } \\
\text { constante actualización de las } \\
\text { temáticas? }\end{array}$ & $\begin{array}{l}\text { Estas preguntas buscan conocer un } \\
\text { poco del conocimiento experiencia } \\
\text { del docente }\end{array}$ \\
\hline $\begin{array}{l}\text { ¿Cómo relaciona el currículo y los } \\
\text { lineamientos curriculares para sus } \\
\text { clases? } \\
\text { ¿Planea su clase? ¿Lleva registro } \\
\text { físico de la planeación? ¿Cómo es } \\
\text { la planeación? } \\
\text { ¿Utiliza material de apoyo en sus } \\
\text { clases? ¿Cuáles? } \\
\text { ¿Cuáles son las temáticas en } \\
\text { ciencia que más le gusta } \\
\text { enseñar? } \\
\text { ¿Qué modelo pedagógico } \\
\text { considera que aplica en sus } \\
\text { clases? }\end{array}$ & $\begin{array}{l}\text { Se realizan con el fin de conocer las } \\
\text { clases de ciencias y desde donde } \\
\text { están fundamentadas. Conociendo } \\
\text { el conocimiento pedagógico del } \\
\text { docente }\end{array}$ \\
\hline $\begin{array}{l}\text { ¿En sus clases tiene en cuenta la } \\
\text { cotidianidad de los estudiantes? } \\
\text { ¿Qué es el conocimiento } \\
\text { cotidiano? }\end{array}$ & $\begin{array}{l}\text { Estas preguntas son relacionadas } \\
\text { con uno de los tópico del proyecto ya } \\
\text { que indaga acerca del conocimiento } \\
\text { cotidiano del maestro y de los } \\
\text { estudiantes }\end{array}$ \\
\hline $\begin{array}{l}\text { ¿Ha trabajado sobre el } \\
\text { conocimiento escolar? } \\
\text { Defina el conocimiento escolar }\end{array}$ & $\begin{array}{l}\text { Las preguntas buscan conseguir } \\
\text { una concepción acerca de la } \\
\text { construcción del conocimiento en } \\
\text { ciencias, si es reconocido por los } \\
\text { docentes o no }\end{array}$ \\
\hline $\begin{array}{l}\text { ¿Cómo relaciona el conocimiento } \\
\text { científico con el conocimiento } \\
\text { cotidiano de sus estudiantes en } \\
\text { las clases de ciencias naturales? }\end{array}$ & $\begin{array}{l}\text { Esta última pregunta busca pruebas } \\
\text { frente a la construcción del } \\
\text { conocimiento en ciencias }\end{array}$ \\
\hline
\end{tabular}

Tabla 1 Aspectos a indagar en la entrevista

Otra herramienta utilizada fue el cuestionario, el cual según Paramo (2008) es una de las herramientas de recolección de información más utilizada en la investigación social ya que a través de este se pueden reunir gran cantidad de datos sobre actitudes, intereses, opiniones, conocimiento, comportamiento, la 
Memorias del 1 Congreso Nacional de Investigación en Enseñanza de la

Biología. VI Encuentro Nacional de Investigación en Enseñanza de la Biología y la Educación Ambiental. ISSN 2027 1034. P. p. 471-478.

ventaja de los cuestio0nrios es su capacidad de recoger datos sobre un amplio rango de posibilidades de respuestas. Por lo anterior es escogido para la recolección de dato en los estudiantes de 601 con el fin de conocer acerca de los gustos, afines e intereses que presentan los estudiantes frente al aprendizaje de las ciencias, las preguntas del cuestionario son sencillas directas y concretas que espera obtener información que permita captar actitudes científicas de los estudiantes además de la idea de construcción de conocimiento que presenta (ver tabla 2)

\begin{tabular}{|c|c|}
\hline Preguntas & Aspecto a indagar \\
\hline $\begin{array}{l}\text { Nombre: } \\
\text { Curso: } \\
\text { Edad: } \\
\text { Género: } \\
\text { Su núcleo familiar está } \\
\text { compuesto por: } \\
\text { Barrio }\end{array}$ & $\begin{array}{l}\text { En esta sección la encuesta } \\
\text { pretende obtener datos frente al } \\
\text { contexto y la realidad social de los } \\
\text { estudiantes de } 601 \text { con el fin de } \\
\text { obtener información frente a los } \\
\text { aspectos socio-económico } \\
\text { familiares de los estudiantes y } \\
\text { poder estimar un acercamiento al } \\
\text { contexto social al que pertenecen }\end{array}$ \\
\hline $\begin{array}{l}\text { ¿Para ti que son las ciencias } \\
\text { naturales? } \\
\text { ¿Cuál es el área que más te gusta } \\
\text { de las ciencias naturales? } \\
\text { ¿Qué elementos te gustaría que } \\
\text { se utilizaran en clase de ciencias } \\
\text { naturales? } \\
\text { ¿Te gusta tu clase de ciencias } \\
\text { naturales? }\end{array}$ & $\begin{array}{l}\text { Estas preguntas se diseñaron con } \\
\text { el fin de indagar el interés de los } \\
\text { estudiantes frente a las ciencias } \\
\text { naturales, con el fin de desarrollar } \\
\text { clases motivantes para los } \\
\text { estudiantes. }\end{array}$ \\
\hline $\begin{array}{l}\text { ¿Crees que los experimentos en } \\
\text { ciencias solo la pueden realizar } \\
\text { los científicos? } \\
\text { ¿Crees que en ciencias son } \\
\text { importantes las constantes } \\
\text { prácticas de laboratorio? }\end{array}$ & $\begin{array}{l}\text { Establece actitudes científicas de } \\
\text { los estudiantes y además permite } \\
\text { establecer la importancia del } \\
\text { conocimiento científico en su } \\
\text { formación. }\end{array}$ \\
\hline $\begin{array}{l}\text { ¿Crees que aprender ciencias } \\
\text { naturales es de alguna utilidad en } \\
\text { tu vida cotidiana? } \\
\text { ¿Aplicas las ciencias naturales } \\
\text { en tu vida cotidiana? } \\
\text { ¿Cómo crees que se relaciona lo } \\
\text { que ves en clases con tu vida? }\end{array}$ & $\begin{array}{l}\text { Nos brinda información acerca de la } \\
\text { construcción del conocimiento } \\
\text { escolar que han venido realizando } \\
\text { los estudiantes en el aérea de } \\
\text { ciencias, realizando la articulación } \\
\text { entre el conocimiento científico y el } \\
\text { cotidiano }\end{array}$ \\
\hline
\end{tabular}

Tabla 2 Aspectos a indagar en el cuestionario 
Bio-grafía Escritos sobre la Biología y su Enseñanza.

Edición Extra-Ordinaria.

Memorias del 1 Congreso Nacional de Investigación en Enseñanza de la

Biología. VI Encuentro Nacional de Investigación en Enseñanza de la Biología y la Educación Ambiental. ISSN 2027 1034. P. p. 471-478.

\section{BIBLIOGRAFÍA}

- BROMME, R. (1988). Conocimientos profesionales de los profesores. Enseñanza de las Ciencias, 6 (1), 19-29.

- HERIQUEZ, R;(2008) Desarrollo del pensamiento científico e investigativo en estudiantes de básica secundaria, Magisterio № 35,82 87

- LLANO, A;(2008). Un alto en el camino ¿científico o católico a la vez? EL tiempo.com, publicado el 3 de Mayo de 2008

- MAZZITELLI, C. APARICIO, M. Las actitudes de los alumnos hacia las ciencias naturales, en el marco de las representaciones sociales, y su influencia en el aprendizaje, Revista Electrónica de Enseñanza de las Ciencias Vol.8 №1 (2009)

- PARAMO, (2008), Técnicas de recolección de información. la investigación en las ciencias sociales. Universidad piloto de Colombia, segunda edición. Bogotá DC

- PEREZ F, (2005) La entrevista como técnica de investigación social, fundamentos teóricos, técnicos y metodológicos. Extramuros v.8 n.22 Caracas mayo

- POZO, J. GÓMEZ, M.(1998) Aprender y enseñar ciencia del conocimiento cotidiano al conocimiento científico, La elaboración del conocimiento científico, Edición Morata

- RODRIGO, M. El hombre de la calle, el científico y el alumno: ¿un solo constructivismo o tres? Novedades educativas № 76 Pág. 59 Abril 1997

- ROBLEDO J, (2009) Observación participante: El acceso al campo, Nure Investigación, n 40, Mayo - Junio.

- ROBLEDO J, (2009) Observación participante: ¿técnica o método? Nure Investigación, ํo 40, Mayo - Junio.

- VALBUENA USSA, Línea de investigación Conocimiento Profesional del Profesor de Ciencias. Vol 2, No 2 (2009) Revista Bio-grafia, Departamento De Biología. Universidad pedagógica nacional . 